\title{
Review of: "Duplication of NRAMP3 gene in poplars generated two homologous transporters with distinct functions"
}

\author{
Omar Pantoja ${ }^{1}$ \\ 1 Universidad Nacional Autónoma de México
}

Potential competing interests: The author(s) declared that no potential competing interests exist.

The work of Pottier et al. reports the characterization of two metal transporters from the NRAMP family from Populus trichocarpa that, interestingly, are located in the same chromosome 7 in a tandem array, with a sequence identity at the amino acid level close to $88 \%$. Both proteins can transport metals in a heterologous expression system, yeast. Although both genes express in all plant organs, the expression of PotriNRAMP3.2 in leaves and stems is sixfold higher. Intracellular location differs between the two paralogs, with PotriNRAMP3.2 showing the same tonoplast localization described for other orthologs, while PotriNRAMP3.1 was found in puncta located at the TGN, indicating a clear distinction in the functioning of these proteins. PotriNRAMP3.2 rescued the Arabidopsis nramp3nramp4 double mutant growth defects under iron starvation, but not PotriNRAMP3.1, a result that agrees with the tonoplast localization of PotriNRAMP3.2, involved in Fe retrieval from the vacuole that is required for seedling development. To assess the physiological role of the two transporters, the authors generated $P$. trichocarpa PotriNRAMP3 overexpressing lines observing several modifications for the PotriNRAMP3.1 lines, like a smaller size and zonal chlorosis between the leaf veins, while subtle changes were observed for the PotriNRAMP3.2 lines. The chlorosis effects observed for PotriNRAMP3.1 were assigned to a higher stem accumulation of $\mathrm{Mn}$, which correlated with a lower $\mathrm{Mn}$ leaf content and a decrease in photosynthesis activity (PSII).

The results in the report are clear and well presented and partially supporting the conclusions given by the authors, however, it might be possible that alternative conclusions can be reached. The main concern are the results obtained from the OE lines and the interpretation given to them. By volume, OE of a tonoplast located transporter, PotriNRAMP3.2, would be expected to have a stronger effect in Mn accumulation than that located at the membrane of an organelle, PotriNRAMP3.1, which could suggest that the changes caused by the OE of PotriNRAMP3.1 may be affecting other processes, rather than preventing Mn release/exocytosis from the vascular tissues to the leaves. Moreover, with a sixfold higher expression, the vacuolar retrieval by PotriNRAMP3.2 should be stronger and therefore, with more effects in Mn stem sequestration and more severe chlorosis in the leaf. In addition, location of PotriNRAMP3.1 to the TGN does not necessarily implies its direct participation in Mn secretion and could indicate its involvement in alternative mechanisms, that by locating to the TGN, may work as a Mn sensor, where several essential 
manganese sugar transferases are housed (Luk E, Culotta VC. J Biol Chem 2001;276:47556; Durr G, Strayle J, Plemper R, Elbs S, Kless SK, Catty P, Wold DH, Rudolph HK. Mol Biol Cell1998;9:1149; Romero PA, Lussier M, Sdicu AM, Bussey H, Herscovics A. Biochem J 1997;321:289). I would suggest that the authors explore alternative explanations to their results in view of the role that $\mathrm{Mn}$ has on the regulation of oxidative stress.

It would be interesting to analyze the protein expression levels both, in the stem and the leaves, taking advantage of the different GFP reporter lines that the authors have generated, which could also be used to quantify protein levels with Western blot analysis. These analyzes could help to support the higher $\mathrm{Mn}$ accumulation observed in the stems in comparison to the leaves. 